http://jmscr.igmpublication.org/home/ ISSN (e)-2347-176x ISSN (p) 2455-0450 crossref DOI: https://dx.doi.org/10.18535/jmscr/v8i6.92

\title{
Chediak-Higashi Syndrome with HLH - A Rare Case Report
}

\author{
Authors \\ Dr Sunil Kumar Agarwalla', Dr B. Maheswar Rao ${ }^{2 *}$, Dr Krishna Dash ${ }^{3}$ \\ ${ }^{1}$ Associate Professor Pediatrics, MKCG MCH, Berhampur, Odisha \\ ${ }^{2}$ Assistant Professor Pediatrics, MKCG MCH, Berhampur, Odisha \\ ${ }^{3}$ Junior Resident Pediatrics, MKCG MCH, Berhampur, Odisha. \\ *Corresponding Author \\ Dr B. Maheswar Rao
}

\begin{abstract}
Chediak Higashi Syndrome (CHS) is a rare autosomal recessive disorder with fewer than 500 cases published worldwide over the last 20 years ${ }^{[1]}$. The important clinical clue for recognising this entity is, by the presence of partial oculo cutaneous albinism. This disease usually presents with recurrent infections and uncommonly also presents with dangerous life threatening condition as HLH (Hemophagocytic Lymphohistiocytosis). The clinical features of this syndrome include partial albinism, photosensitivity, severe recurrent bacterial infections, bleeding diathesis, and late onset neurological manifestations (central and peripheral neuropathy, sensory loss, muscle weakness, cerebellar ataxia, and cognitive impairment $)^{[2,3]}$. Approximately $85 \%$ of cases develop a fatal accelerated phase (HLH) characterized by pancytopenia, hemophagocytosis, and marked infiltration of organs by lymphocytes leading to hepatosplenomegaly, lymphadenopathy and finally with multi-organ dysfunction $(M O D S)^{[6]}$.

Owing to the rarity of the condition and the characteristic clinical and hematological findings, we report a case of Chediak-Higashi Syndrome (CHS) which presented with HLH.

Keywords: Chediak Higashi, partial albinism, HLH, MODS.
\end{abstract}

\section{Introduction}

Chediak Higashi Syndrome is a rare autosomal recessive, complex immune disorder that usually occurs in childhood characterized by oculocutaneous albinism. The decrease in phagocytosis results in recurrent pyogenic infection and a tendency to bruise and bleed easily.

\section{Case Report}

A 2.5y old girl, 1st order, born out of third degree consanguineous marriage presented with high grade fever, swelling of both sides of neck, progressive pallor, loss of appetite and abdominal distension for 20 days. Never hospitalised before for any major illness, had mild ARI 3 episodes in the past. Fully immunised as per NIS, neurodevelopmentally normal child. No family history of similar illness. On examination child was febrile, irritable, weighing $10 \mathrm{~kg}$ with features of silvery hair (blond hair) mixed with black hair, hypopigmented eye lashes and eyebrows, light brown coloured iris without any photophobia or nystagmus. Some pallor present, no icterus, 
cyanosis, clubbing, oedema or any bleeding. Presence of significant bilateral cervical and axillary lymphadenopathy which were nontender, non matting. The abdomen was protruberent with massive hepatosplenomegaly. The respiratory system, cardiovascular system and nervous system revealed no abnormality.
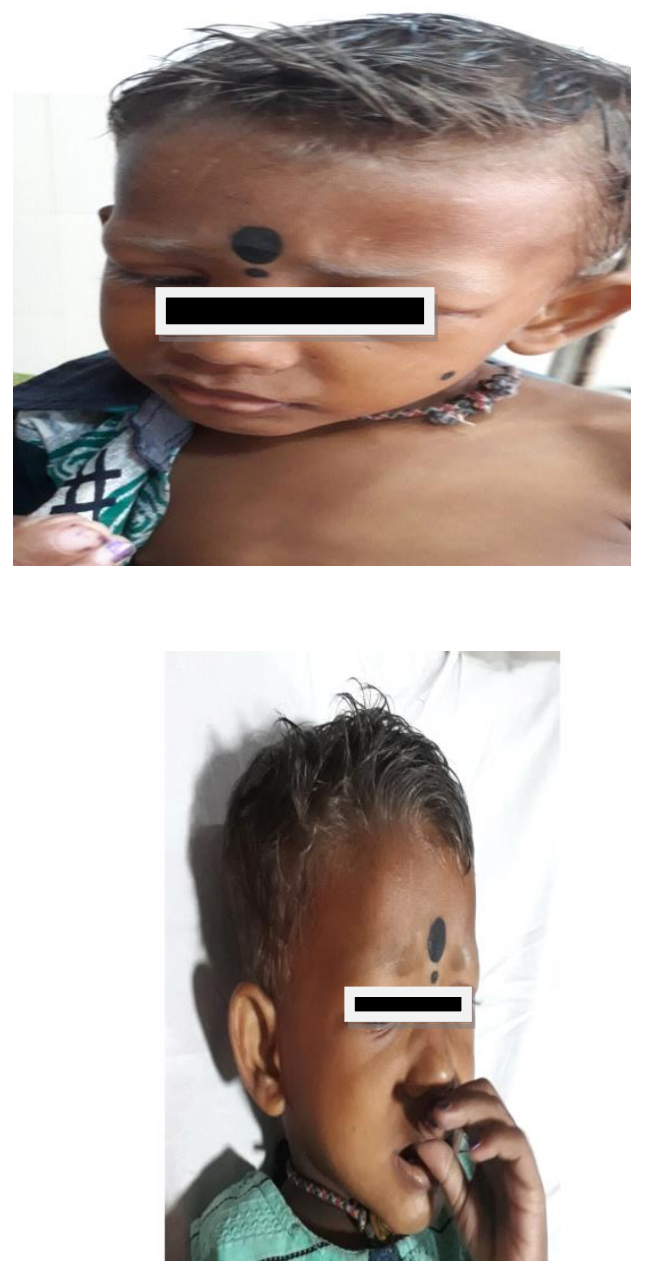

Pic $1 \& 2$ shows hypopigmented blond hair of scalp, eyelashes and eyebrow]

The laboratory investigations revealed anaemia $\mathrm{Hb} \quad 6.7 \mathrm{gm} / \mathrm{dl}, \quad$ TWBC $13.50 \times 10^{9} / \mathrm{L}, \quad$ TPC $58 \times 10^{9} / \mathrm{L}$ and peripheral smear showed microcytic hypochromic anaemia with thrombocytopenia with few atypical cells.

MP ICT - Negative, Widal Test - Negative, Sickling Test - negative, Scrub typhus IgM Elisa - negative, HIV - negative, Mantouex Test Negative. CXR normal, USG abdomen showed only hepatosplenomegaly.
High Serum ferritin - $2000 \mathrm{ng} / \mathrm{ml}$, high serum triglyceride level - $1613 \mathrm{mg} / \mathrm{dl}$. Bone marrow aspiration cytology showed evidence of haemophagocytosis and myelopoiesis accelerated with myelocytes and metamyelocytes showing giant toxic granules, erythropoiesis and megakaryopoiesis being normal.

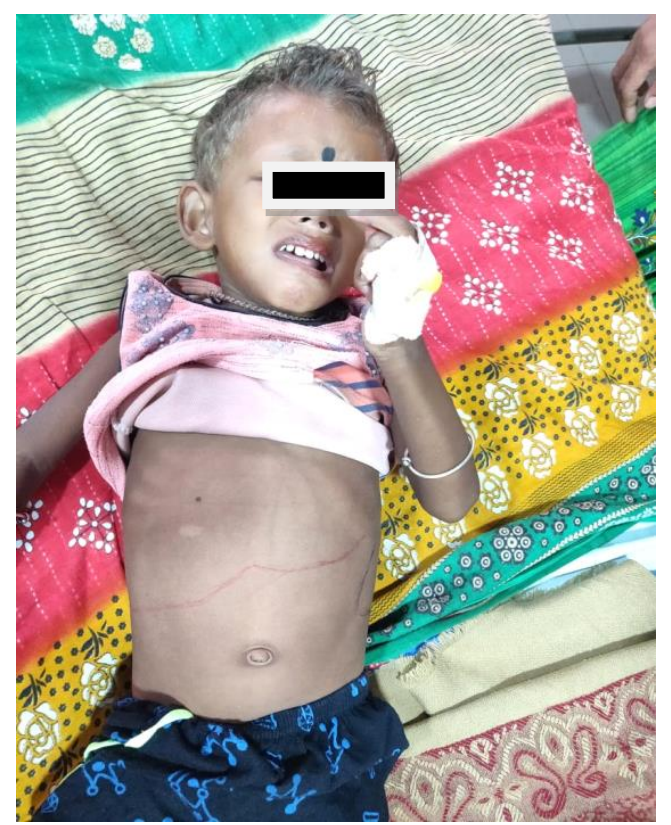

Pic.3 Showing huge hepatosplenomegaly

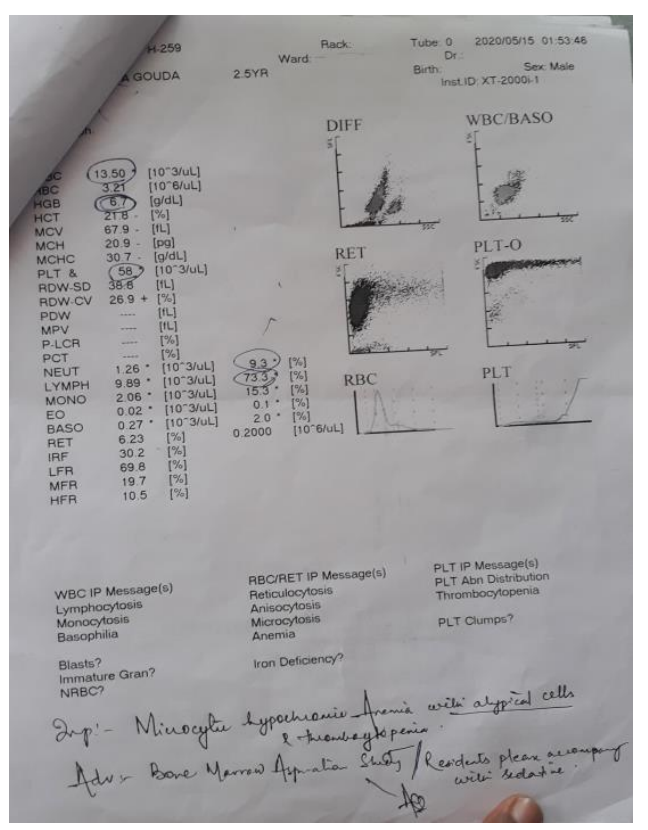

Pic.4 CBC Shows Bicytopenia

The patient fulfilled the diagnostic criteria for hemophagocytic lymphohistiocytosis (HLH) as she had prolonged fever, splenomegaly, bicytopenia, high ferritin level, 
hypertriglyceridemia, and hemophagocytosis. Thus, the diagnosis of accelerated phase of CHS was made on the basis of clinical presentation of partial oculocutaneous albinism (blond hair, hypopigmented eyelashes and eyebrows, light brown coloured iris ) and hematological findings of giant granules in myelocytes and metamyelocytes of bone marrow.

Blood and urine cultures were normal, no evidence of Tuberculosis found. Serum bilirubin being 1.6 (T) \& 0.9 (D), SGOT 185, SGPT 56, normal serum creatinine $0.7 \mathrm{mg} / \mathrm{dl}$. The function of NK cell activity was not feasible in our setup.

The child was initially treated with inj. Ceftriaxone, oral Azithromycin and oral fluconazole for $5 \mathrm{~d}$ but there was no improvement of the patient. After diagnosis of HLH the child was put on inj. Dexamethasone and inj. Etoposide and there was dramatic improvement within 5 days as evidenced by absence of fever, almost absent lymphnodes and decrease in size of liver and spleen and appearance of general well being with return of appetite.

\section{Discussion}

Chediak - Higashi Syndrome, the name is derived from a Cuban Hematologist - Chediak and Japanese Pediatrician - Higashi, who described a series of cases with similar features and manifestations. CHS is a rare disease (approximately 500 cases reported worldwide), the prevalence and incidence of which are unknown. In a nationwide survey in Japan, 15 patients were diagnosed during a period of 11 years (2000-2010), indicating that one or two patients with CHS were diagnosed each year ${ }^{[4]}$.

CHS is characterized by partial oculocutaneous albinism, repeated infections, and pathognomonic abnormal giant granules in neutrophils, lymphocytes, monocytes, and platelets. Patients develop recurrent infections that most commonly involve the skin and respiratory system. Staphylococcus aureus and beta-hemolytic Streptococcus are the predominant organisms. Viral and fungal infections, however, have also been described ${ }^{[5]}$. Increased susceptibility to recurrent infections is attributed to defects in $\mathrm{T}$ cell cytotoxicity and natural killer (NK) function and defects in granulocyte chemotaxis and bactericidal activity ${ }^{[6]}$.

The accelerated phase is observed in $85 \%$ of individuals and can occur at any age, including shortly after birth or within several years. Clinical manifestations include fever, lymphadenopathy, hepatosplenomegaly, anemia, neutropenia, thrombocytopenia, and neurological abnormalities $^{[7]}$. CHS can also present with mild bleeding diathesis due to platelet aggregation defect. It can also present with neuropathy and ataxia, which usually begins in teenage.

Our case was a challenge for the diagnosis, initially we ruled out malaria, sickle cell disease, tuberculosis and scrub typhus after specific investigation. But the degree of hepatosplenomegaly made us to think in the line of leukemia or HLH. Presence of atypical cells provoked us to think in line of leukemia. The important pointer or the clue to our diagnosis was the presence of partial albinism.

Roy et al. (2011) studied the clinicohematological profile of five cases of CHS, reporting that all patients had silvery hair, partial albinism, photophobia, and recurrent skin and/or chest infection. Three of them $(50 \%)$ presented with accelerated phase (HLH). Prognosis associated with the accelerated phase was poor ${ }^{[8]}$.

The genetic hallmark of CHS is mutations in the LYST / CHS gene located on chromosome 1q2q44 [9]. Mutations of this gene result in a defect in granule morphogenesis in multiple tissues ${ }^{[10]}$. The gene encodes a protein called the lysosomal traffick regulator which regulates the synthesis, transport, and fusion of cytoplasmic vesicles ${ }^{[11]}$. The abnormalities observed in these vesicles result in grossly enlarged and nonfunctional lysosomes, which are identified during cytology as giant coalesced azurophilic granules present mostly in granulocytes and monocytes, but also in fibroblasts, melanocytes, astrocytes, Schwann cells, and hematopoietic cells ${ }^{[11]}$. These granules 
are specific to CHS and their presence in granulocytes from peripheral blood and bone marrow is the basis of diagnosis ${ }^{[10]}$.

The only treatment that cures the hematologic and immunologic defects is allogenic hematopoietic stem cell transplantation (HSCT), but this therapy does not prevent the progressive neurological dysfunction frequently observed during long-term follow up ${ }^{[1,5]}$.

The current standard of care is HSCT as soon as the diagnosis is confirmed and the accelerated phase has either been ruled out or is in remission. The most favourable outcome is achieved when HSCT is performed prior to development of the accelerated phase. If signs of the accelerated phase are present, hemophagocytosis must be brought into clinical remission before HSCT can be performed. Guidelines for treatment of the accelerated phase, revised in $2004^{[12]}$, are the same as those for familial hemophagocytic lymphohistiocytosis (fHLH). Combination therapy consists of etoposide, dexamethasone, and cyclosporine A. Remission is achieved in $75 \%$ of individuals within 8 weeks ${ }^{[13]}$. However, relapses are common and response to treatment declines over time. Once remission occurs, prompt HSCT is recommended.

\section{Conclusion}

CHS is a rare disease with a varied spectrum of clinical presentation and investigation findings. The partial oculo-cutaneous albinism is the clue for thinking about this rare entity. The prognosis of the accelerated phase is poor. HSCT is the only curative treatment for hematological and immunological disorders. We emphasize the need for early diagnosis on basis of characteristic clinical findings and diagnostic laboratory examinations, which leads to early HSCT before development of the accelerated phase.

Though Partial ALBINISM can be familial but with FUO, massive hepatosplenomegaly one has to think of Chediak Higashi Syndrome.
Conflicts of interest: The authors declare no conflicts of interest.

\section{References}

1. Kaplan J, De Domenico I, Ward DM. Chediak-Higashi syndrome. Curr Opin Hematol 2008;15(1):22-9.

2. De Azambuja AP, DoNascimento B, Comar SR, Loth G, Ribeiro LL, Bonfim C, et al. Four cases of Chediak-Higashi syndrome. Rev Bras Hematol Hemoter 2011;33:315-22.

3. Bhambhani $\mathrm{V}$, Introne $\mathrm{WJ}$, Lungu $\mathrm{C}$, Cullinane A, Toro C. Chediak-Higashi syndrome presenting as young-onset levodopa- responsive parkinsonism. Mov Disord 2013;28:127-9.

4. Nagai K, Ochi F, Terui K, Maeda M, Ohga $\mathrm{S}$, Kanegane $\mathrm{H}$, et al. Clinical characteristics and outcomes of ChediakHigashi syndrome: a nationwide survey of Japan. Pediatr Blood Cancer 2013;60: 1582-6.

5. Dotta L, Parolini S, Prandini A, Tabellini A, Antolini M, Kurtzberg J, et al. Clinical, laboratory and molecular signs of immunodeficiency in patients with partial oculo-cutaneous albinism. Orphanet J Rare Dis 2013;8:168.

6. Pujani M, Agarwal K, Bansal S, Ahmad I, Puri V, Verma D, et al. Chediak-Higashi syndrome: a report of two cases with unusual hyperpigmentation of the face. Turk Patoloji Derg 2011;27:246-8.

7. Usha HN, Prabhu PD, Sridevi M, Baindur K, Balakrishnan CM. Chediak-Higashi syndrome. Indian Pediatr 1994;34:1115-9.

8. Roy A, Kar R, Basu D, Srivani S, Badhe BA. Clinico-hematological profile of Chediak Higashi: experience from a tertiary care center in south India. Indian J Pathol Microbiol 2011;54:547-51.

9. Barrat FJ, Auloge L, Pastural E, Lagelouse RD, Vilmer E, Cant AJ, et al. Genetic and physical mapping of the Chediak- Higashi 
syndrome on chromosome 1q42-43. Am J Hum Genet 1996;59:625-32.

10. De Azambuja AP, DoNascimento B, Comar SR, Loth G, Ribeiro LL, Bonfim C, et al. Four cases of Chediak-Higashi syndrome. Rev Bras Hematol Hemoter 2011;33:315-22.

11. Olkkonen VM, Ikonen E. Genetic defects of intracellularmembrane transport. N Engl J Med 2000;343:1095-104.

12. Henter JI, Horne A, Arico' M, Egeler RM, Filipovich AH,Imashuku S, et al. HLH 2004: diagnostic and therapeutic guidelines for hemophagocytic lymphohistiocytosis. Pediatr Blood Cancer 2007;48:124-31.

13. Filipovich AH. Hemophagocytic lymphohistiocytosis and related disorders. Curr Opin Allergy Clin Immunol 2006;6: 410-5. 